\title{
Model Selection in Iterative Valuation QUESTIONS
}

\author{
by \\ Emmanuel Flachaire \\ Université Paris 1 Panthéon-Sorbonne \\ and \\ Guillaume Hollard \\ Université de Marne la Vallée
}

July 2007

\begin{abstract}
In this article, we propose a unified framework that accomodates many of the existing models for dichotomous choice contingent valuation with follow-up and allows to discriminate between them by simple parametric tests of hypothese. Our empirical results show that the Range model, developped in Flachaire and Hollard (2007), outperforms other standard models and confirms that, when uncertain, respondents tend to accept proposed bids.
\end{abstract}

Key words: starting point bias, preference uncertainty, contingent valuation JEL Classification: Q26, C81 


\section{Introduction}

The NOAA panel recommends the use of a dichotomous choice format in contingent valuation (CV) surveys (Arrow et al. 1993). To improve the efficiency of dichotomous choice contingent valuation surveys, follow-up questions are frequently used. While these enhance the efficiency of dichotomous choice surveys, several studies have found that they yield willingness-to-pay estimates that are substantially different from estimates implied by the first question alone. This is the so-called starting point bias. ${ }^{1}$ Many authors have proposed some specific models to handle this problem (Herriges and Shogren 1996, Alberini et al. 1997, DeShazo 2002, Whitehead 2002, Cooper et al. 2002, Lechner et al. 2003, Flachaire and Hollard 2006).

In Flachaire and Hollard (2007), we proposed a model, called the Range model, in which individuals hold a range of acceptable values, rather than a precisely defined value of their willingness-to-pay. ${ }^{2}$ In the Range model, starting point bias occurs as a result of respondent uncertainty when answering the first question, while existing models assume that starting point bias occurs while answering the second question. ${ }^{3}$

This paper proposes further tests of the Range model: (1) we test the Range model on another dataset and (2) we test the Range model against most existing models. An additional result of this paper is a clarification of the relation among existing models. It is shown that existing models can be derived from three general ones. In some favorable cases, this allows us to compare the performance of existing models.

The article is organized as follows. The following section presents the Range model. The subsequent sections present other standard models, the interrelation between all the models and an application. The final section concludes.

\section{$2 \quad$ Range model}

The Range model, developed in Flachaire and Hollard (2007), is a dichotomous choice model which explains starting point bias by respondent's uncertainty. It models the individual decision process, using the principle of "coherent arbitrariness" (Ariely et al. 2003), ${ }^{4}$ and can be estimated from a bivariate probit model.

\footnotetext{
${ }^{1}$ Other response effects could explain the difference between estimates of mean WTP, as framing, respondents assumptions about the scope of the program and wastefulness of the government, see Alberini et al. 1997 for a dicussion.

${ }^{2}$ This is in line with studies putting forward that individuals are rather unsure of their own willingness-to-pay (Li and Mattsson 1995, Ready et al. 1995, 2001, Welsh and Poe 1998, van Kooten et al. 2001, Hanley and Kriström 2002, Alberini et al. 2003).

${ }^{3} \mathrm{~A}$ notable exception is Lechner et al. 2003.

${ }^{4}$ These authors conducted a series of valuation experiments. They observed that "preferences are initially malleable but become imprinted (i.e. precisely defined and largely invariant) after the individual is called upon to make an initial decision".
} 


\subsection{Decision process}

In dichotomous choice contingent valuation with follow-up questions, two questions are presented to respondents. The first question is "Would you agree to pay $\mathrm{x} \$$ ?". The second, or follow-up, question is similar but asks for a higher bid offer if the initial answer is yes and a lower bid offer otherwise. The Range model is based on the following decision process :

1. Prior to a valuation question, the respondent holds a range of acceptable values:

$$
w t p_{i} \in\left[\underline{W}_{i}, \bar{W}_{i}\right] \quad \text { with } \quad \bar{W}_{i}-\underline{W}_{i}=\delta
$$

where $\bar{W}_{i}$ is the upper bound of the range.

2. Confronted with a first valuation question, the respondent selects a value inside that range according to the following rule:

$$
W_{i}=\operatorname{Min}_{w t p_{i}}\left|w t p_{i}-b_{1 i}\right| \quad \text { with } \quad w t p_{i} \in\left[\underline{W}_{i}, \bar{W}_{i}\right]
$$

A respondent selects a value so as to minimize the distance between his range of willingness-to-pay and the proposed bid $b_{1 i}$. In other words, $W_{i}=b_{1 i}$ if the bid falls within the WTP range, $W_{i}$ is equal to the upper bound of the range if $b_{1 i}$ is greater than the upper bound of the WTP range, and $W_{i}$ is equal to the lower bound of the range if $b_{1 i}$ is less than the lower bound of the WTP range.

3. The respondent answers the questions according to the selected value:

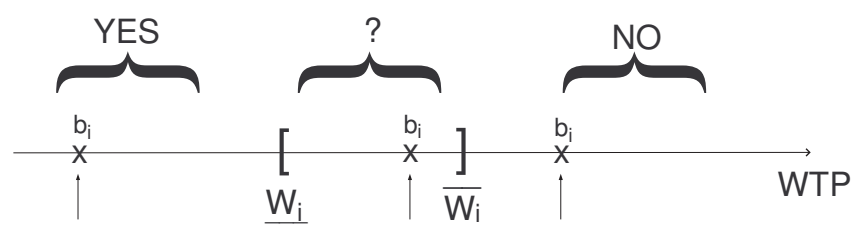

He will agree to pay any amount below $\underline{W}_{i}$ and refuse to pay any amount that exceeds $\bar{W}_{i}$. When the first bid falls within the WTP range, he can answer yes or no (?): we assume in such case that a respondent answers yes to the first question with a probability $\xi$ and no with a probability $1-\xi$.

If respondents always answer yes when the first bid belongs to the interval of acceptable

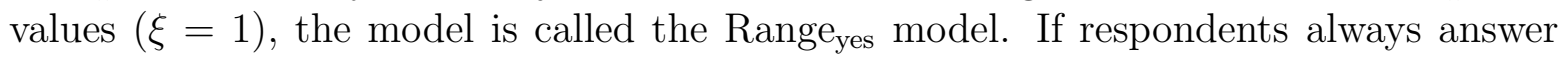
no when the first bid belongs to the interval of acceptable values $(\xi=0)$, the model is called the Range no $_{\text {model. }}$ 


\subsection{Estimation}

In Flachaire and Hollard (2007), we show that the Range model can be estimated from a more general random effect probit model, that also encompasses the Shift model proposed by Alberini et al. (1997). If we use a linear model and if we assume that the distribution of WTP is Normal, the probability that the individual $i$ answers yes to the $j^{\text {th }}$ question, $j=1,2$ equals to:

$$
\mathcal{M}_{1}: \quad P\left(W_{j i}>b_{j i}\right)=\Phi\left[X_{i} \alpha-\frac{1}{\sigma} b_{j i}+\lambda_{1} D_{j} r_{1 i}+\lambda_{2} D_{j}\left(1-r_{1 i}\right)\right]
$$

where $r_{1 i}$ is the response to the first payment question, $D_{1}=0$ and $D_{2}=1, \alpha=\beta / \sigma$, $\lambda_{1}=\delta_{1} / \sigma$ and $\lambda_{2}=\delta_{2} / \sigma$. Based on this equation, the parameters are interrelated according to:

$$
\beta=\alpha \sigma, \quad \delta_{1}=\lambda_{1} \sigma \quad \text { and } \quad \delta_{2}=\lambda_{2} \sigma .
$$

When we use just the responses to the initial payment question $(j=1)$, this equation simplifies to:

$$
P(y e s)=P\left(W_{1 i}>b_{1 i}\right)=\Phi\left[X_{i} \alpha-\frac{1}{\sigma} b_{1 i}\right]
$$

Moreover, the probability that the individual $i$ answers yes to the initial and the followup questions $\left(r_{1 i}=1, j=2\right)$ is equal to:

$$
P(y e s, y e s)=\Phi\left[X_{i} \alpha-\frac{1}{\sigma} b_{2 i}+\frac{\delta_{1}}{\sigma}\right]
$$

From the estimation based on $\mathcal{M}_{1}$, different models can be considered:

- $\delta_{1}<0$ and $\delta_{2}>0$ corresponds to the Range model (with $\delta_{2}-\delta_{1}=\delta$ ).

- $\delta_{1}<0$ and $\delta_{2}=0$ corresponds to the Range yes $_{\text {model }}$

- $\delta_{1}=0$ and $\delta_{2}>0$ corresponds to the Range no $_{\text {model }}$

- $\delta_{1}=\delta_{2}$ corresponds to the Shift model

- $\delta_{1}=\delta_{2}=0$ corresponds to the Double-bounded model.

It is clear that the Range model and the Shift model are non-nested (one model is not a special case of the other); they can be tested through $\mathcal{M}_{1}$.

\subsection{Interpretation}

Estimation of the Range model provides estimates of $\beta, \sigma, \delta_{1}$ and $\delta_{2}$, from which we can estimate a mean of WTP $\mu_{\xi}$ and a dispersion of WTP $\sigma$. This last mean of WTP would 
be similar to the mean of WTP estimated using the first questions only, that is, based on the single-bounded model. Additional information is obtained from the use of follow-up questions: estimates of $\delta_{1}$ and $\delta_{2}$ allow us to estimate a range of means of WTP:

$$
\left[\mu_{0} ; \mu_{1}\right]=\left[\mu_{\xi}+\delta_{1} ; \mu_{\xi}+\delta_{2}\right] \quad \text { with } \quad \delta_{1} \leq 0, \quad \text { and } \quad \delta_{2} \geq 0 .
$$

The lower bound $\mu_{0}$ corresponds to the case where respondents always answer no if the bid belongs to the range of acceptable values $(\xi=0)$. Conversely, the upper bound $\mu_{1}$ corresponds to the case where respondents always answer yes if the bid belongs to the range of acceptable values $(\xi=1)$. How respondents answer the question when the bid belongs to the range of acceptable values can be tested as follows:

- respondents always answer no corresponds to the null hypothesis $H_{0}: \delta_{1}=0$

- respondents always answer yes corresponds to the null hypothesis $H_{0}: \delta_{2}=0$.

\section{Interrelation with standard models}

Different models are proposed in the literature to control for starting point bias: anchoring bias, structural shift effects and ascending/descending sequences. All these models assume that the second answer is sensitive to the first bid offer. They assume that a prior willingness-to-pay $W_{i}$ is used to answer the first bid offer, and an updated willingness-topay $W_{i}^{\prime}$ is used by the respondents to answer the second bid. It follows that an individual answers yes to the first and to the second bids if:

$$
r_{1 i}=1 \quad \Leftrightarrow \quad W_{i}>b_{1 i} \quad \text { and } \quad r_{2 i}=1 \quad \Leftrightarrow \quad W_{i}^{\prime}>b_{2 i}
$$

Each model leads to a specific definition of $W_{i}^{\prime}$. In the following subsections, we briefly review some standard models, their estimation and the possible interrelations between them and the range model previously defined.

\subsection{Models}

Anchoring model: Herriges and Shogren (1996) propose a model where the respondents combine their prior WTP with the value provided by the first bid as follows:

$$
W_{i}^{\prime}=(1-\gamma) W_{i}+\gamma b_{1 i}
$$

The first bid offer plays the role of an anchor: it causes the WTP to come to it.

Shift model: Alberini et al. (1997) propose a model where the WTP systematically shifts between the two answers:

$$
W_{i}^{\prime}=W_{i}+\delta
$$


The first bid offer is interpreted as providing information about the cost or the quality of the object. Indeed, a respondent can interpret a higher bid offer as paying more for the same object and a lower bid offer as paying less for a lower quality object.

Anchoring \& Shift model: Whitehead (2002) proposes a model that combines anchoring and shift effects:

$$
W_{i}^{\prime}=(1-\gamma) W_{i}+\gamma b_{1 i}+\delta
$$

In addition, see Aadland and Caplan (2004) and Whitehead (2004) for estimation details.

Framing model: DeShazo (2002) proposes de-constructing iterative questions into their ascending and descending sequences. His results show that the answers that follow an initial yes cause most of the problems. He recommends using the decreasing follow-up questions only:

$$
W_{i}^{\prime}=W_{i} \quad \text { if } \quad r_{1 i}=0
$$

Using prospect theory (Kahneman and Tversky 1979), Deshazo argues that the first bid offer is interpreted as a reference point if the answer to the first question is yes: the follow-up question is framed as a loss and the respondents are more likely to answer no to the second question.

Framing $\&$ Anchoring $\&$ Shift model: Flachaire and Hollard (2006) propose applying anchoring and shift effects in ascending sequences only:

$$
W_{i}^{\prime}=W_{i}+\gamma\left(1-W_{i}\right) r_{1 i}+\delta r_{1 i}
$$

It takes into account questions that follow an initial yes. Empirical results suggest that gains in efficiency can be obtained compared to the Framing model. Note that this model is not based on the underlying decision process defined in section 2 .

\subsection{Estimation}

Implementation of the Anchoring $\&$ Shift model can be based on a random effect probit model, with the probability that the individual $i$ answers yes to the $j^{\text {th }}$ question, $j=1,2$ equals to:

$$
\mathcal{M}_{2}: \quad P\left(W_{j i}>b_{j i}\right)=\Phi\left[X_{i} \alpha-\frac{1}{\sigma} b_{j i}+\theta\left(b_{1 i}-b_{j i}\right) D_{j}+\lambda D_{j}\right]
$$

where $D_{1}=0$ and $D_{2}=1, \alpha=\beta / \sigma, \theta=\gamma /(\sigma-\gamma \sigma)$ and $\lambda=\delta /(\sigma-\gamma \sigma)$. Based on this equation, the parameters are interrelated according to:

$$
\beta=\alpha \sigma, \quad \gamma=\theta \sigma /(1+\theta \sigma) \quad \text { and } \quad \delta=\lambda \sigma(1-\gamma) .
$$

Implementation of the Anchoring model and of the Shift model can be derived from this last probability, respectively with $\delta=0$ and $\gamma=0$. The Double-bounded model corresponds to the case $\delta=\gamma=0$. 
The Framing 83 Anchoring $\&$ Shift model differs from the previous model by the fact that anchoring and shift effects occur in ascending follow-up questions only. Its implementation can be based on a random effect probit model, with the probability that the individual $i$ answers yes to the $j^{\text {th }}$ question, $j=1,2$ equals to:

$$
\mathcal{M}_{3}: \quad P\left(W_{j i}>b_{j i}\right)=\Phi\left[X_{i} \alpha-\frac{1}{\sigma} b_{j i}+\theta\left(b_{1 i}-b_{j i}\right) D_{j} r_{1 i}+\lambda D_{j} r_{1 i}\right]
$$

where $D_{1}=0$ and $D_{2}=1, \alpha=\beta / \sigma, \theta=\gamma /(\sigma-\gamma \sigma)$ and $\lambda=\delta /(\sigma-\gamma \sigma)$. Based on this equation, the parameters are interrelated according to (15).

\subsection{Interrelation between all the models}

It can be helpful to see the interrelations between all the models. Indeed, some models are nested and thus, we can test a restricted model against an unrestricted model with standard inference based on a null hypothesis. Table 1 shows the restrictions to apply

\begin{tabular}{|c||c|c|c|}
\hline Model & $\mathcal{M}_{1}$ & $\mathcal{M}_{2}$ & $\mathcal{M}_{3}$ \\
\hline \hline Double & $\delta_{1}=\delta_{2}=0$ & $\gamma=\delta=0$ & $\gamma=\delta=0$ \\
\hline Anchoring & & $\delta=0$ & \\
\hline Shift & $\delta_{1}=\delta_{2}$ & $\gamma=0$ & \\
\hline Anch-Shift & & n. c. & \\
\hline Fram-Anch-Shift & & & n. c. \\
\hline Range & $\delta_{1} \leq 0 \leq \delta_{2}$ & & \\
\hline Range yes $_{\text {An }}$ & $\delta_{1} \leq 0, \delta_{2}=0$ & & $\gamma=0$ \\
\hline
\end{tabular}

Table 1: Nested Models (n.c.: no constraints)

to the probabilities $\mathcal{M}_{1}, \mathcal{M}_{2}$ and $\mathcal{M}_{3}$, defined in equations (3), (14), (16), in order to estimate the different models. For instance, it is clear that the Shift and the Range models are non-nested, but they are both special cases of $\mathcal{M}_{1}$. Thus, a Shift model can be selected against a Range model through the general form $\mathcal{M}_{1}$.

\section{Application}

In this application, we use a survey that involves a sample of users of the natural reserve of the Camargue, a major wetland in the south of France. The purpose of the contingent valuation survey was to evaluate how much individuals were willing to pay as an entrance fee to contribute to the preservation of the natural reserve. The survey was administered to 218 recreational visitors during spring 1997, using face to face interviews. Recreational visitors were selected randomly in seven sites all around the natural reserve. The WTP 
question used in the questionnaire was a dichotomous choice with follow-up. ${ }^{5}$ For a complete description of the contingent valuation survey, see Claeys-Mekdade, Geniaux, and Luchini (1999). Mean values of the WTP were estimated using a linear model (McFadden and Leonard 1993). Indeed, Crooker and Herriges (2004) show that the simple linear probit model is often more robust in estimating the mean WTP than other parametric and semi-parametric models.

The mean and the dispersion of WTP estimated from a single bounded model are:

$$
\begin{array}{lrl}
\hat{\mu}=113.5 & \text { with a confidence interval } & \text { [98.1;138.2] } \\
\hat{\sigma}=45.3 & \text { with a standard error } & 17.9
\end{array}
$$

The confidence interval of $\hat{\mu}$ is obtained by simulation with the Krinsky and Robb procedure, see Haab and McConnell (2003, ch.4) for more details.

Let us consider the following standard models: double-bounded, anchoring, shift, anchoring $\&$ shift models. These models can be estimated from $\mathcal{M}_{2}$, with or without some specific restrictions, see (14). Table 2 presents estimated means of WTP $\hat{\mu}$ and the dispersion of WTP distributions $\hat{\sigma}$. Standard errors are given in italics and confidence intervals of $\hat{\mu}$ are presented in brackets; they are obtained by simulation with the Krinsky

\begin{tabular}{|c|c|c|c|c|c|c|c|c|c|c|c|c|}
\hline \multirow[t]{2}{*}{$\mathcal{M}_{2}$} & \multirow[t]{2}{*}{ constraint } & \multicolumn{2}{|c|}{ mean WTP } & \multicolumn{2}{|c|}{ disp WTP } & \multicolumn{2}{|c|}{ anchor } & \multicolumn{2}{|c|}{ shift } & \multicolumn{2}{|c|}{ corr. } & \multirow[b]{2}{*}{$\ell$} \\
\hline & & $\mu$ & c.i. & $\sigma$ & s.e. & & s.e. & $\delta$ & s.e. & & s.e. & \\
\hline Double & $\gamma=\delta=0$ & 89.8 & {$[84.4 ; 96.5]$} & 52.6 & 10.0 & - & & - & & 0.71 & 0.16 & -177.3 \\
\hline Anchoring & $\delta=0$ & 133.8 & {$[108.4 ; 175.2]$} & 92.0 & 44.5 & 0.51 & 0.23 & - & & 0.78 & 0.14 & -175.2 \\
\hline Shift & $\gamma=0$ & 119.4 & [105.7;139.7] & 69.0 & 19.9 & - & & -26.7 & 9.1 & 0.63 & 0.17 & -173.1 \\
\hline Anch-Shift & n. c. & 158.5 & [122.6;210.7] & 100.8 & 53.5 & 0.46 & 0.29 & -17.1 & 13.9 & 0.73 & 0.16 & -172.1 \\
\hline
\end{tabular}
and Robb procedure.

Table 2: Random effect probit models estimated from $\mathcal{M}_{2}$

As expected, the confidence interval of the mean WTP and the standard error of the dispersion of the WTP decrease significantly when we use the usual double-bounded model (Double) instead of the previous single-bounded model. However, estimates of the mean WTP in both models are very different (89.8 vs. 113.5). Such inconsistent results suggest a problem of starting-point bias. It leads us to consider the Anchoring 8 Shift model (Anch-Shift) to control for such effects, in which the Double, Anchoring and Shift models are nested. We can compute a likelihood-ratio statistic $(L R)$ to test a restricted model against the Anchoring 8 Shift model. The $L R$ test is twice the difference between the maximized value of the loglikelihood functions (given in the last column), which is asymptotically distributed as a Chi-squared distribution. We can test the Double model against the Anch-Shift model with the null hypothesis $H_{0}: \gamma=\delta=0$, for which $L R=10.4$. A $P$-value can be computed and is equal to $P=0.0055$ : we reject

\footnotetext{
${ }^{5}$ The first bid $b_{1 i}$ is drawn randomly from $\{5,10,15,20,25,30,35,40,45,50,60,70,80,90,100\}$. If the answer to the first bid is $n o$, a second bid $b_{2 i}<b_{1 i}$ is drawn randomly. If the answer to the first bid is yes, a second bid $b_{2 i}>b_{1 i}$ is drawn randomly. There was a high response rate $(92.6 \%)$.
} 
the null hypothesis and thus the Double model. We can test the Anchoring model against the Anch-Shift model $\left(H_{0}: \delta=0\right)$ : we reject the null hypothesis $(P=0.0127)$. Finally, we can test the Shift model against the Anch-Shift model $\left(H_{0}: \gamma=0\right)$ : we do not reject the null $(P=0.1572)$. From this analysis, the Shift model is selected.

It is interesting to observe that, when we compare the results between the Shift and the Single-bounded models, the confidence intervals and standard errors are not significantly different. This supports the conclusion of Herriges and Shogren (1996): they argue that once we have controlled for the starting-point effect, the efficiency gains from the follow-up questioning can be small.

To go further, we consider a model where anchoring and shift effects occur in ascending sequences, but not in descending sequences (Fra-Anc-Shi). The case with shift effect in ascending sequences (no anchoring) corresponds to the Range model where respondents always answer yes if the initial bid belongs to their range of acceptable values.

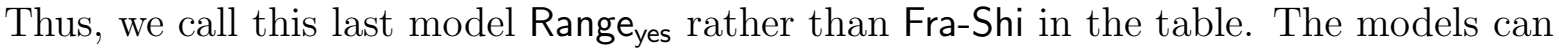
be estimated from $\mathcal{M}_{3}$, with or without some specific restrictions, see (16). Estimation results are given in Table 3.

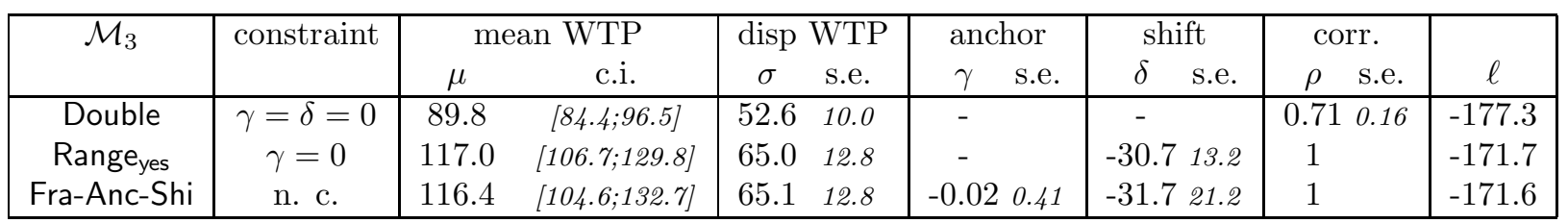

Table 3: Random effect probit models estimated from $\mathcal{M}_{3}$

If we compute a LR statistic to test the Double model against the Fra-Anc-Shi model $\left(H_{0}: \gamma=\delta=0\right)$, we reject the null hypothesis $(P=0.0033)$. Conversely, if we test the Range $_{\text {yes }}$ model against the Fra-Anc-Shi model $\left(H_{0}: \gamma=0\right)$, we do not reject the null

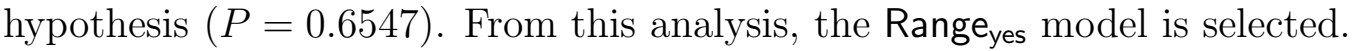

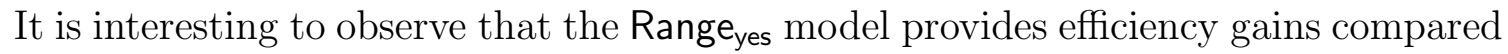
to the single-bounded and Shift models: confidence intervals and standard errors of the mean and of the dispersion of WTP are smaller. However, the Shift model is selected

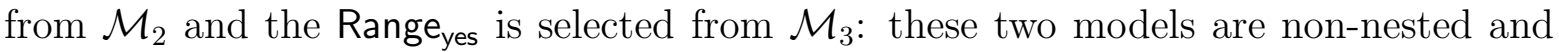
no inference is used to select one model.

Next, we consider the model developed in this article, that considers starting pointbias with respondent's uncertainty. This model can be estimated from a more general model $\mathcal{M}_{1}$ and corresponds to the case $\delta_{1} \leq 0 \leq \delta_{2}$, see (3). An interesting feature of $\mathcal{M}_{1}$ is that the Double and the Shift model are special cases, respectively with the restrictions $\delta_{1}=\delta_{2}=0$ and $\delta_{1}=\delta_{2}$. Thus, even if the Range and the Shift models are non-nested, we can test them through $\mathcal{M}_{1}$. Estimation results are given in Table 4 . The estimation result, obtained with no restrictions, provides $\hat{\delta}_{1} \leq 0 \leq \hat{\delta}_{2}$. It corresponds to the case of the Range model and thus, estimation results with no constraints are presented in the line called Range. This result suggests that the Range model is more 


\begin{tabular}{|c|c|c|c|c|c|c|c|c|c|c|c|c|}
\hline \multirow[t]{2}{*}{$\mathcal{M}_{1}$} & \multirow[t]{2}{*}{ constraint } & \multicolumn{2}{|c|}{ mean WTP } & \multicolumn{2}{|c|}{ disp WTP } & \multicolumn{2}{|c|}{ shift $_{1}$} & \multicolumn{2}{|c|}{ shift $_{2}$} & \multicolumn{2}{|c|}{ corr. } & \multirow[b]{2}{*}{$\ell$} \\
\hline & & $\mu$ & c.i. & $\sigma$ & s.e. & & s.e. & $\delta_{2}$ & s.e. & & s.e. & \\
\hline Double & $\delta_{1}=\delta_{2}=0$ & 89.8 & {$[84.4 ; 96.5]$} & 52.6 & 10.0 & - & & - & & 0.71 & 0.16 & -177.3 \\
\hline Shift & $\delta_{1}=\delta_{2}$ & 119.4 & [105.7;139.7] & 69.0 & 19.9 & -26.7 & 9.1 & -26.7 & 9.1 & 0.63 & 0.17 & -173.1 \\
\hline Range & n. c. & 126.0 & {$[110.7 ; 147.3]$} & 73.5 & 21.6 & -43.7 & 27.6 & 6.5 & 8.8 & 1 & & -171.5 \\
\hline Range $_{\text {yes }}$ & $\delta_{1} \leq 0, \delta_{2}=0$ & 117.0 & [106.7;129.8] & 65.0 & 12.8 & -30.7 & 13.2 & - & & 1 & & -171.7 \\
\hline
\end{tabular}

Table 4: Random effect probit models estimated from $\mathcal{M}_{1}$

appropriate than the Shift model, otherwise we would have had $\delta_{1}$ and $\delta_{2}$ quite similar and with the same sign. This can be confirmed by testing the Shift model against the Range model $\left(H_{0}: \delta_{1}=\delta_{2}\right)$; we reject the null hypothesis $(P=0.0736)$ at a nominal

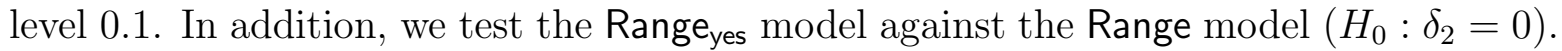
We do not reject the null hypothesis $(P=0.5270)$. From this analysis, the Range yes model is selected.

Finally, inference based on $\mathcal{M}_{1}, \mathcal{M}_{2}$ and $\mathcal{M}_{3}$ leads us to select a Range model, where the respondents answers yes if the initial bid belongs to their range of acceptable values $^{6}$. This model gives an estimator of the mean WTP close to the single-bounded model (117.0 vs. 113.5) with a smaller confidence interval ([106.7;129.8] vs. [98.1;138.2]) and smaller standard errors (12.8 vs. 17.9). Table 5 presents full econometric results of this model with the single-bounded model. It is clear from this table that the standard errors in the Range yes are always significantly reduced compared to the standard errors in the single-bounded model. In other words, the selected Range model provides both consistent results with the single-bounded model and efficiency gains. Furthermore, we can draw additional information from the Range model. Indeed, from (7) we have:

$$
\left[\hat{\mu}_{0} ; \hat{\mu}_{1}\right]=[86.3 ; 117] \quad \text { and } \quad \hat{\delta}=30.7
$$

This model provides a range of values, rather than a unique WTP mean value.

From our results, we can make a final observation. Estimation of a random effect probit model with an estimated correlation coefficient $\rho$ less than unity suggests that respondents use two different values of WTP to answer the first and the second questions. This is a common interpretation in empirical studies; see Alberini et al. (1997). If we restrict our analysis to the standard models (Double, Anchoring, Shift and Anch-Shift), our results leads us to select the Shift model, for which $\hat{\rho}=0.63$ (significantly less than 1). However, if we consider a more general model $\mathcal{M}_{1}$ that encompasses the Range and the Shift models, estimation results leads us to select the Range yes $_{\text {model for which } \hat{\rho}=1}$ (the estimation does not restrict the parameter $\rho$ to be equal to one, this estimated value equals to one is obtained from an unrestricted estimation). It suggests that respondents

\footnotetext{
${ }^{6}$ The Range $_{\text {yes }}$ model is empirically equivalent to a special case developed in Flachaire and Hollard (2006), with an anchoring parameter equal to zero. In this last article, the results suggested a specific behavior (shift effect) in ascending sequences only. This interpretation was based on empirical results only, with an unknown underlying decision process. Here, we obtain similar empirical results, but the interpretation of the response behavior is very different.
} 


\begin{tabular}{lcccc}
\hline \multirow{2}{*}{ Variables } & \multicolumn{2}{c}{ Single } & \multicolumn{2}{c}{ Range ${ }_{\text {yes }}$} \\
& coef. & s.e. & coef. & s.e. \\
\hline \hline Distance home-natural site & 0.21 & 0.07 & 0.08 & 0.04 \\
Using a car to arrive & -1.36 & 0.66 & -0.94 & 0.34 \\
Employee & 2.11 & 0.67 & 1.07 & 0.39 \\
Middle class & 2.42 & 0.64 & 1.27 & 0.37 \\
Inactive & 1.16 & 0.67 & 0.77 & 0.43 \\
Working class & 2.14 & 0.92 & 0.99 & 0.54 \\
White collars & 1.77 & 0.51 & 1.01 & 0.33 \\
Visiting with family & 0.10 & 0.50 & 0.16 & 0.34 \\
Visiting alone & 1.35 & 1.01 & 1.49 & 0.66 \\
Visiting with a group & 0.99 & 0.73 & 0.07 & 0.43 \\
First visit & 1.13 & 0.46 & 0.22 & 0.22 \\
New facilities proposed & 1.25 & 0.39 & 0.67 & 0.20 \\
Other financing proposed & -0.70 & 0.34 & -0.35 & 0.19 \\
South-West & -0.53 & 0.56 & -0.51 & 0.39 \\
South-East & 0.93 & 0.72 & 0.59 & 0.44 \\
Questionnaire type & -0.62 & 0.31 & -0.20 & 0.19 \\
Investigator 1 & 0.52 & 0.72 & 0.13 & 0.45 \\
Investigator 2 & -0.38 & 0.77 & -0.43 & 0.49 \\
$b_{1}$ & -0.02 & 0.01 & -0.02 & 0.01 \\
Constant & 0.78 & 0.99 & 1.24 & 0.68 \\
\hline
\end{tabular}

Table 5: Full econometric estimation results

answer both questions according to the same value, contrary to the results obtained with the standard models.

\section{Conclusion}

In this article, we propose a unified framework that accomodates many of the existing models for dichotomous choice contingent valuation with follow-up and allows to discriminate between them by simple parametric tests of hypothese. We further test the Range model, developped in Flachaire and Hollard (2007), against several others standard models. Our empirical results show that the Range model outperforms other standard models and that, when uncertain, respondents tend to accept proposed bids. It confirms that respondent uncertainty is a valid explanation of various anomalies arising in contingent valuation surveys.

\section{References}

Aadland, D. and A. J. Caplan (2004). "Incentive incompatibility and starting-point bias in iterative valuation questions: comment". Land Economics 80(2), 312-315. 
Alberini, A., K. Boyle, and M. Welsh (2003). "Analysis of contingent valuation data with multiple bids and response options allowing respondents to express uncertainty". Journal of Environmental Economics and Management 45, 40-62.

Alberini, A., B. Kanninen, and R. Carson (1997). "Modeling response incentive effects in dichotomous choice valuation data". Land Economics 73(3), 309-324.

Ariely, D., G. Loewenstein, and D. Prelec (2003). "Coherent arbitrariness: Stable demand curves without stable preferences". Quarterly Journal of Economics 118-1, 73-105.

Arrow, K., R. Solow, P. R. Portney, E. E. Leamer, R. Radner, and H. Schuman (1993, January). Report of the NOAA panel on contingent valuation. Technical Report 58(10), p1601-1614.

Claeys-Mekdade, C., G. Geniaux, and S. Luchini (1999). Quelle valeur attribuer à la Camargue? Une perspective interdisciplinaire économie et sociologie. In P. Point (Ed.), La valeur économique des hydrosystèmes. Méthodes et modèles d'évaluation des services délivrés, Chapter 7, pp. 137-168. Paris: Economica.

Cooper, J., W. M. Haneman, and G. Signorelli (2002). "One and one-half bids for contingent valuation". Review of Economics and Statistics 84, 742-750.

Crooker, J. R. and J. A. Herriges (2004). "Parametric and semi-nonparametric estimation of willingness-to-pay in the dichotomous choice contingent valuation framework". Environmental and Resource Economics 27, 451-480.

DeShazo, J. R. (2002). "Designing transactions without framing effects in iterative question formats". Journal of Environmental Economics and Management 43, p360-385.

Flachaire, E. and G. Hollard (2006). "Controlling starting-point bias in double-bounded contingent valuation surveys". Land Economics 82, 103-111.

Flachaire, E. and G. Hollard (2007). "Starting-point bias and respondent uncertainty in dichotomous choice valuation surveys". Resource and Energy Economics, forthcoming.

Haab, T. C. and K. E. McConnell (2003). Valuing Environmental and Natural Resources. Edwar Elgar.

Hanley, N. and B. Kriström (2002). "What's it worth? Exploring value uncertainty using interval questions in contingent valuation". working paper, 2002-10, University of Glasgow.

Herriges, J. A. and J. F. Shogren (1996). "Starting point bias in dichotomous choice valuation with follow-up questioning". Journal of Environmental Economics and Management 30, 112-131.

Kahneman, D. and A. Tversky (1979). "Prospect theory: an analysis of decisions under risk". Econometrica 47, 313-327.

Lechner, S., A. Rozan, and F. Laisney (2003). "A modelisation of the anchoring effect in closed-ended question with follow-up". Document de travail du BETA, 2003-07.

Li, C. Z. and L. Mattsson (1995). "Discrete choice under preference uncertainty: an improved structural model for contingent valuation". Journal of Environmental Economics and Management 28, 256-269. 
McFadden, D. and G. Leonard (1993). Issues in the contingent valuation of environmental goods: Methodologies for data collection and analysis. In Contingent Valuation: a critical Assessment, pp. 165-215. New York: North-Holland: Hausman.

Ready, R. C., S. Navrud, and W. R. Dubourg (2001). "How do respondents with uncertain willingness to pay answer contingent valuation questions?". Land Economics 77, 315-326.

Ready, R. C., J. C. Whitehead, and G. Blomquist (1995). "Contingent valuation when respondents are ambivalent". Journal of Environmental Economics and Management 29, 181-196.

van Kooten, G. C., E. Krcmar, and E. H. Bulte (2001). "Preference uncertainty in non-market valuation: a fuzzy approach". American Journal of Agricultural Econmics 83, 487-500.

Welsh, M. P. and G. L. Poe (1998). "Elicitation effects in contingent valuation: comparisons to a multiple bounded discrete choice approach". Journal of Environmental Economics and Management 36, 170-185.

Whitehead, J. C. (2002). "Incentive incompatibility and starting-point bias in iterative valuation questions". Land Economics 78, p285-297.

Whitehead, J. C. (2004). "Incentive incompatibility and starting-point bias in iterative valuation questions: reply". Land Economics 80(2), 316-319. 